\title{
MicroRNA-30a increases the chemosensitivity of U251 glioblastoma cells to temozolomide by directly targeting beclin 1 and inhibiting autophagy
}

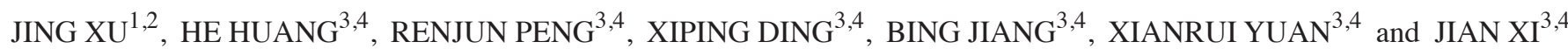 \\ ${ }^{1}$ Department of Otolaryngology-Head and Neck Surgery, Xiangya Hospital of Central South University; \\ ${ }^{2}$ The Institute of Skull Base Surgery and Neurooncology at Hunan; ${ }^{3}$ The Neurosurgical Institute of Central South University; \\ ${ }^{4}$ Department of Neurosurgery, Xiangya Hospital of Central South University, Changsha, Hunan 410008, P.R. China
}

Received December 2, 2015; Accepted September 22, 2016

DOI: $10.3892 /$ etm.2018.6007

\begin{abstract}
Temozolomide (TMZ) is one of the most commonly used drugs for the clinical treatment of glioblastomas. However, it has been reported that treatment with TMZ can induce autophagy, which leads to tumor resistance and increases the survival of tumor cells. MicroRNA-30a (miR-30a) has been found to have inhibitory effects on autophagy by directly targeting beclin 1. However, the exact role of miR-30a in TMZ-treated glioblastoma cells has not been studied previously. The present study aimed to investigate whether miR-30a increased the cytotoxicity of TMZ to glioblastoma U251 cells, as well as the underlying mechanism. MTT and flow cytometry assay results showed that treatment with TMZ inhibited the proliferation of $\mathrm{U} 251$ cells while inducing cell apoptosis in a dose-dependent manner. Western blotting data showed that the expression levels of LC3-II and beclin 1 as well as the ratio of LC3-II to LC3-I were markedly increased in TMZ-treated U251 cells compared with the untreated control cells, indicating that treatment with TMZ induced autophagy. Moreover, reverse transcription-quantitative polymerase chain reaction data showed that treatment with TMZ led to a significant reduction in miR-30a levels in a dose-dependent manner in U251 cells. Elevation of the miR-30a level significantly inhibited TMZ-induced autophagy, demonstrated by the decreased LC3-II and beclin 1 levels and ratio of LC3-II to LC3-I, accompanied by the reduced proliferation and increased apoptosis in TMZ-treated U251 cells. Furthermore, luciferase reporter assay data indicated that beclin 1 was a direct target of miR-30a in U251 cells. In summary, this study demonstrated that miR-30a increases the chemosensitivity of glioblastoma
\end{abstract}

Correspondence to: Dr Jian Xi, Department of Neurosurgery, Xiangya Hospital of Central South University, 87 Xiangya Road, Changsha, Hunan 410008, P.R. China

E-mail: doctorxijian@sina.com

Key words: glioblastoma, autophagy, temozolomide, microRNA, chemotherapy resistance
U251 cells to temozolomide by directly targeting beclin 1 and inhibiting autophagy. Therefore, autophagy may be a promising target for the treatment of TMZ-resistant tumors.

\section{Introduction}

Gliomas are the most common brain tumors, accounting for $\sim 30 \%$ of central nervous system tumors and $80 \%$ of all malignant brain tumors (1). Due to the resistance of gliomas to radiotherapy, chemotherapy and adjuvant therapies, the median survival rate of glioblastomas and high grade gliomas has not been markedly improved over the past few decades (2-5). Currently, temozolomide (TMZ) is one of the most commonly used drugs for the clinical treatment of glioblastomas (6). $\mathrm{TMZ}$ can inhibit the proliferation of cancer cells via the induction of cell cycle arrest, and induce tumor cell apoptosis $(7,8)$. However, there is evidence indicating that the administration of TMZ also induces tumor chemotherapy resistance via the induction of autophagy.

Autophagy, an evolutionarily conserved function, is a cellular self-catabolic degradation process, responsible for the lysosomal degradation of long-lived proteins as well as aged or damaged organelles (9). The amino acids and fatty acids generated during autophagy can be reused and thus autophagy may be of benefit for sustainable cell survival (10). A number of studies have strongly suggested that autophagy is activated in cancer cells under certain chemotherapy treatments, including TMZ, leading to tumor chemotherapy resistance (11-13). Moreover, inhibition of autophagy has been found to enhance the efficacy of TMZ therapy in glioblastomas $(14,15)$.

MicroRNAs (miRs), a class of non-coding RNAs, 18-25 nucleotides in length, are able to induce mRNA degradation or suppress protein translation via binding to the 3'-untranslated regions (3'-UTRs) of mRNA of specific genes (16). Moreover, miRs have been demonstrated to be involved in a variety of cellular processes, including proliferation, survival, differentiation, maturation and apoptosis, as well as autophagy $(17,18)$. Among these miRs, miR-30a has been found to play a suppressive role in autophagy via the direct targeting of beclin 1, a key autophagy-promoting gene that is critical in the regulation of cell survival and death (19). 
In addition, miR-30a-induced inhibition of autophagy has been reported to sensitize certain tumor cells to chemotherapy (20). For instance, Zou et al found that miR-30a sensitized tumor cells to cisplatin via the suppression of beclin 1-mediated autophagy (21). Yu et al reported that inhibition of autophagy mediated by miR-30a enhanced imatinib activity against human chronic myeloid leukemia cells (22). However, to the best of our knowledge, the detailed role of miR-30a in the regulation of TMZ-induced autophagy has never been reported in glioblastomas.

In the present study, the aim was to investigate whether miR-30a has an effect on TMZ-induced autophagy in glioblastomas. In addition, the involvement of beclin 1 in the underlying molecular mechanism was explored.

\section{Materials and methods}

Cell culture. Human glioblastoma U251 cells were obtained from the China Cell Culture Center (Shanghai, China). The U251 cells were cultured in Dulbecco's modified Eagle's medium (DMEM; Thermo Fisher Scientific, Inc., Waltham, MA, USA) supplemented with $10 \%$ fetal bovine serum (FBS), $100 \mathrm{U} / \mathrm{ml}$ penicillin and $100 \mathrm{mg} / \mathrm{ml}$ streptomycin (all from Thermo Fisher Scientific, Inc.). To mimic chemotherapy, U251 cells were treated with TMZ $(1,5,10$ or $30 \mu \mathrm{g} / \mathrm{ml})$ for $6 \mathrm{~h}$, and then examined by a series of assays.

Reverse transcription-quantitative polymerase chain reaction (RT-qPCR) analysis. TRIzol Reagent (Thermo Fisher Scientific, Inc.) was used to extract total RNA from U251 cells, in accordance with the manufacturer's instructions. A RevertAid First Strand cDNA Synthesis kit (Fermentas; Thermo Fisher Scientific, Inc., Pittsburgh, PA, USA) was used to reverse transcribe total RNA into cDNA, according to the manufacturer's protocol. The miRNA expression was determined using a PrimeScript ${ }^{\circledR}$ miRNA RT-PCR kit (Takara Biotechnology Co., Ltd., Dalian, China), in accordance with the manufacturer's instructions. The PCR conditions were $95^{\circ} \mathrm{C}$ for $10 \mathrm{~min}$, and 40 cycles of denaturation at $95^{\circ} \mathrm{C}$ for $30 \mathrm{sec}$ and annealing/elongation at $60^{\circ} \mathrm{C}$ for $30 \mathrm{sec}$. The primer sequences for miR-30a were: Forward, 5'-GGGGTGTAA ACATCCTCGACTG-3' and reverse, 5'-ATTGCGTGTCGT GGAGTCG-3'. The primer sequences for U6 were: Forward, 5'-GCTTCGGCAGCACATATACTAAAAT-3' and reverse, 5'-CGCTTCACGAAT TTGCGTGTCAT-3'. They were purchased from Shanghai GenePharma Co., Ltd. (Shanghai, China). All miRNA data are expressed relative to a U6 small nuclear RNA from the same sample. Independent experiments were repeated three times. The relative expression levels of mRNA were analyzed by use of the $2^{-\Delta \Delta \mathrm{Cq}}$ method (23).

Transfection. Lipofectamine 2000 (Thermo Fisher Scientific, Inc.) was used to perform transfection according to the manufacturer's protocol. Briefly, U251 cells were cultured to $70 \%$ confluence, and resuspended in serum-free DMEM. Serum-free DMEM was used to dilute Lipofectamine 2000, miR-30a mimic, or scrambled miR mimic, respectively. The diluted Lipofectamine 2000 was then added to the diluted miR-30a mimic or diluted scrambled miR mimic. After incubation for $20 \mathrm{~min}$ at room temperature, the mixture was added to the cell suspension. After incubation at $37^{\circ} \mathrm{C}$ with $5 \% \mathrm{CO}_{2}$ for $6 \mathrm{~h}$, the medium was replaced by DMEM supplemented with $10 \%$ FBS. Following transfection for $48 \mathrm{~h}$, the following assays were performed.

MTT assay. An MTT assay was performed to evaluate the cell proliferation. In brief, $1 \times 10^{4} \mathrm{U} 251$ cells from each group were plated in a 96-well plate, and incubated for 6, 12, 24 and $48 \mathrm{~h}$ at $37^{\circ} \mathrm{C}$ with $5 \% \mathrm{CO}_{2}$. MTT $(5 \mathrm{mg} / \mathrm{ml}$; Thermo Fisher Scientific, Inc.) was then added to each well, and the plate was incubated for $4 \mathrm{~h}$ at $37^{\circ} \mathrm{C}$ with $5 \% \mathrm{CO}_{2}$. The supernatant was removed, and $100 \mu \mathrm{l}$ dimethylsulfoxide (Thermo Fisher Scientific, Inc.) was added to dissolve the precipitate. The absorbance was detected at $492 \mathrm{~nm}$ using the BioTek ${ }^{\mathrm{TM}}$ ELX800 ${ }^{\mathrm{TM}}$ Absorbance Microplate reader (BioTek Instruments, Inc., Winooski, VT, USA).

Cell apoptosis assay. Cell apoptosis was determined using an Annexin V-FITC Apoptosis Detection kit (BD Pharmingen, San Diego, CA, USA), according to the manufacturer's instruction. In brief, U251 cells were harvested and washed with cold PBS twice. After that, U251 cells $\left(1 \times 10^{6}\right)$ were resuspended in $200 \mu \mathrm{l}$ binding buffer with $10 \mu \mathrm{l}$ Annexin-V-FITC and $5 \mu \mathrm{l}$ PI-PE, and incubated in the dark for $30 \mathrm{~min}$. Following incubation, $300 \mu \mathrm{l}$ binding buffer was added and the cells were analyzed by flow cytometry (C6 cytometer; Beckman Coulter, Inc. (Brea, CA, USA).

Western blotting. U251 cells were solubilized in cold radioimmunoprecipitation assay lysis buffer (Thermo Fisher Scientific, Inc.) to extract protein, which was separated by 10\% SDS-PAGE (Pierce; Thermo Fisher Scientific, Inc.), and transferred onto a polyvinylidene difluoride (PVDF) membrane (Pierce). The PVDF membrane was incubated with rabbit anti-LC3-II polyclonal antibody (1:50; ab48394; Abcam, Cambridge, MA, USA), rabbit anti-LC3-I polyclonal primary antibody (1:50; ab128025; Abcam), rabbit anti-beclin 1 monoclonal antibody (1:100; ab55878; Abcam) and rabbit anti-GAPDH polyclonal primary antibody (1:100; ab9485; Abcam), respectively, at $4^{\circ} \mathrm{C}$ overnight. After washing with PBST three times, the PVDF membrane was then incubated with mouse anti-rabbit secondary antibody (1:5,000; ab99697; Abcam) at room temperature for $40 \mathrm{~min}$. Chemiluminescent detection was conducted using an ECL kit (Pierce). The protein expression was analyzed using Image-Pro plus software 6.0 (Media Cybernetics, Inc., Rockville, MD, USA), and the expression levels were represented as the density ratio vs. GAPDH.

Bioinformatic analysis and luciferase reporter gene assay. Targetscan software (version 3.1; targetscan.org/mamm_31/) was used to predict the putative target of miR-30a. The wild type (WT) or mutant type (MUT) 3'-UTR of the beclin 1 gene (BECN1) was obtained from Yearthbio (Qingdao, China), amplified from human genomic DNA and then cloned downstream of the firefly luciferase coding region in the pmirGLO ${ }^{\mathrm{TM}}$ Luciferase vector (Promega Corporation, Madison, WI, USA), to produce pMIR-WT BECN1 and pMIR-MUT BECN1, respectively. After that, U251 cells were co-transfected with pMIR-WT BECN1 or pMIR-MUT 
A

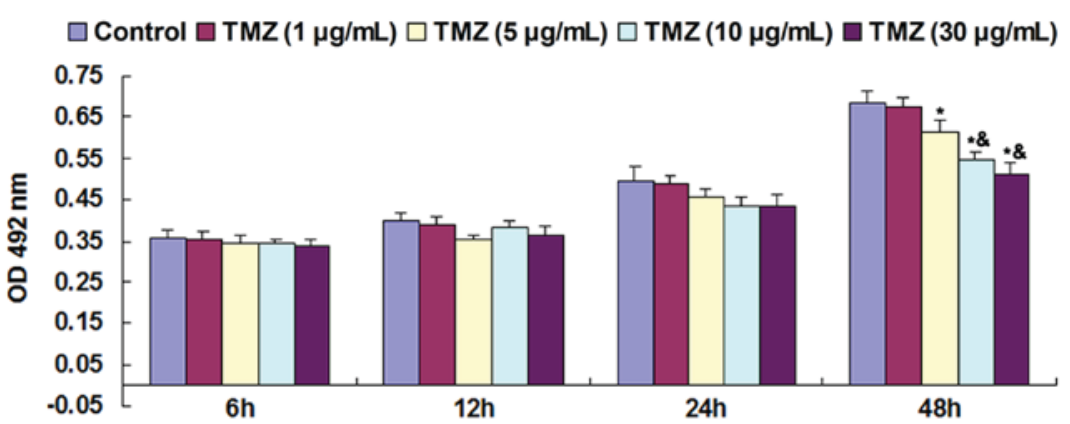

B
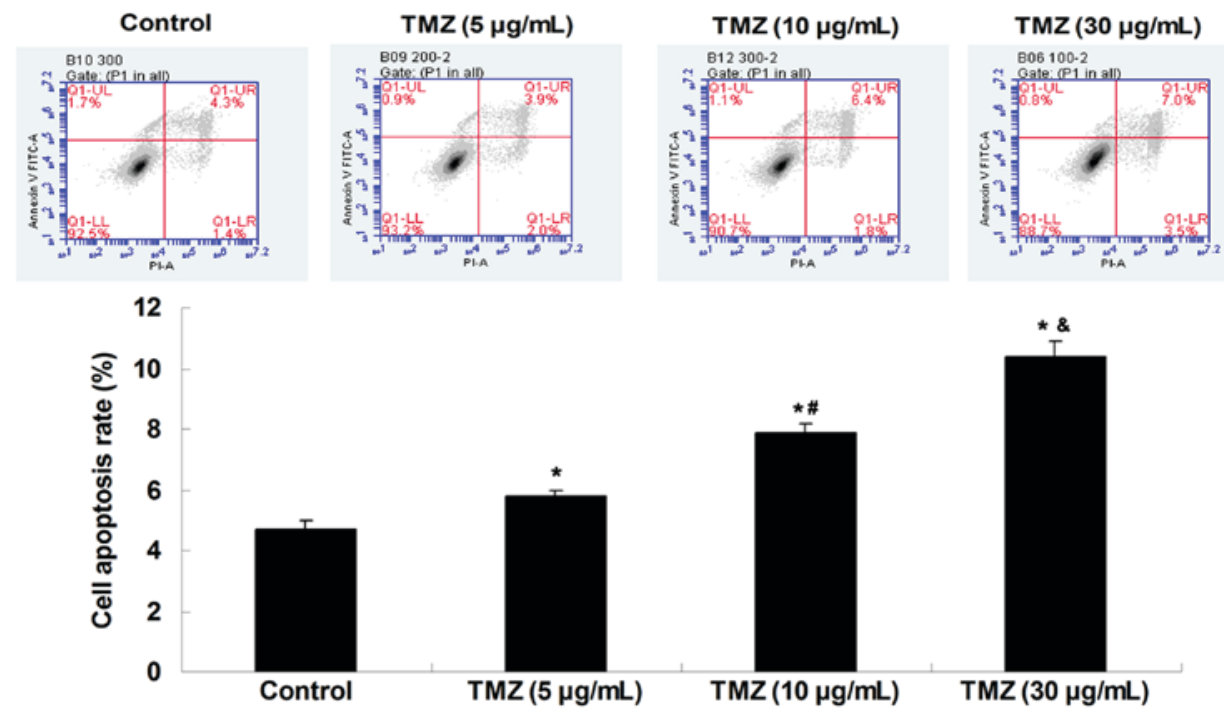

C
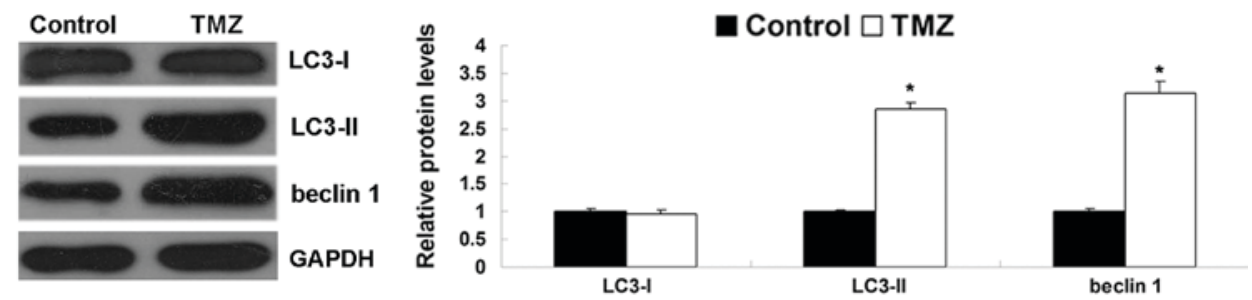

D
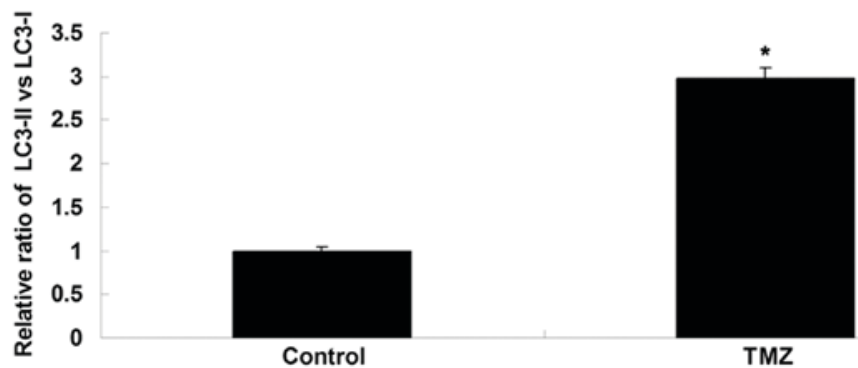

Figure 1. Treatment with TMZ inhibits proliferation, and induces apoptosis and autophagy in U251 cells. (A) MTT assay was performed to examine the proliferation of U251 cells treated with TMZ $(1-30 \mu \mathrm{g} / \mathrm{ml})$. ${ }^{*} \mathrm{P}<0.05$ vs. control. ${ }^{\circledR} \mathrm{P}<0.05 \mathrm{vs}$. TMZ $(1 \mu \mathrm{g} / \mathrm{ml})$. (B) Flow cytometry was performed to examine the apoptosis of U251 cells treated with TMZ (1-30 $\mu \mathrm{g} / \mathrm{ml})$. ${ }^{*} \mathrm{P}<0.05$ vs. control. ${ }^{~} \mathrm{P}<0.05 \mathrm{vs}$. TMZ $(5 \mu \mathrm{g} / \mathrm{ml})$. ${ }^{\circledR} \mathrm{P}<0.05 \mathrm{vs}$. TMZ (10 $\left.\mu \mathrm{g} / \mathrm{ml}\right)$. (C) Western blot analysis was conducted to determine the levels of autophagy-related proteins in $\mathrm{U} 251$ cells treated with TMZ (30 $\mu \mathrm{g} / \mathrm{ml})$. "P<0.05 vs. control. (D) The ratio of LC3-II to LC3-I was calculated in U251 cells treated with TMZ (30 $\mu \mathrm{g} / \mathrm{ml})$. "P<0.05 vs. control. Non-treated U251 cells were used as the control in each assay. TMZ, temozolomide.

BECN1 vector and miR-30a mimic or scrambled miR mimic, and the pRL-TK plasmid (Promega Corporation) for internal normalization, respectively, and cultured for $48 \mathrm{~h}$. The transfected cells were then lysed using lysis buffer (Promega
Corporation). A luciferase reporter gene assay was then conducted using the Dual-Luciferase Reporter Assay system (Promega Corporation), in accordance with the manufacturer's instructions. 


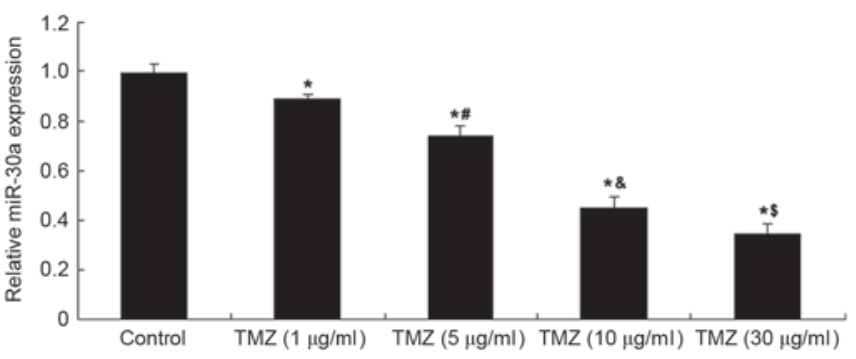

Figure 2. Treatment with TMZ decreases the expression of miR-30a in U251 cells. Reverse transcription-quantitative polymerase chain reaction was conducted to examine the relative miR-30a levels (relative to U6) in U251 cells treated with TMZ (1-30 $\mu \mathrm{g} / \mathrm{ml})$. Non-treated U251 cells were used as a control. ${ }^{*} \mathrm{P}<0.05$ vs. control. ${ }^{\#} \mathrm{P}<0.05$ vs. TMZ $(1 \mu \mathrm{g} / \mathrm{ml}) .{ }^{\&} \mathrm{P}<0.05$ vs. TMZ $(5 \mu \mathrm{g} / \mathrm{ml}) .{ }^{\$} \mathrm{P}<0.05$ vs. TMZ $(10 \mu \mathrm{g} / \mathrm{ml})$. TMZ, temozolomide.

Statistical analysis. All data are represented as the mean of at least triplicate samples \pm standard deviation. Statistical analysis of differences was performed by one-way analysis of variance using SPSS version 17.0 software (SPSS, Inc., Chicago, IL, USA). $\mathrm{P}<0.05$ was considered to indicate a statistically significant difference.

\section{Results}

Treatment with TMZ inhibits proliferation and induces apoptosis and autophagy in U251 cells. U251 cells were treated with TMZ (1-30 $\mu \mathrm{g} / \mathrm{ml})$. Following treatment for 6-48 h, an MTT assay was performed to examine the cell proliferation. As shown in Fig. 1A, the administration of TMZ markedly inhibited U251 cell proliferation in a concentration-dependent manner. As $1 \mu \mathrm{g} / \mathrm{ml}$ of TMZ showed no effect on U251 cell proliferation, this concentration was not used in the following experiments. Subsequently, the effect of TMZ on U251 cell apoptosis was examined. The results indicated that treatment with TMZ $(5,10$ or $30 \mu \mathrm{g} / \mathrm{ml})$ significantly induced U251 cell apoptosis in a concentration-dependent manner (Fig. 1B). Therefore, the highest concentration $(30 \mu \mathrm{g} / \mathrm{ml})$ of TMZ was used when analyzing the effect of TMZ on protein expression. Western blot analysis was conducted to determine the levels of autophagy-related proteins. As shown in Fig. 1C, higher levels of LC3-II and beclin 1 were observed in TMZ-treated U251 cells compared with the control group. Furthermore, the ratio of LC3-II to LC3-I in the U251 cells was also increased following treatment with TMZ (Fig. 1D). These findings indicate that treatment with TMZ inhibits proliferation, while inducing apoptosis and autophagy in U251 glioblastoma cells.

Treatment with TMZ decreases the expression of miR-30a in U251 cells. As miR-30a has been demonstrated to play a suppressive role in autophagy, the expression level of miR-30a in U251 cells with or without treatment with TMZ (1-30 $\mu \mathrm{g} / \mathrm{ml})$ was determined. As shown in Fig. 2, the expression level of miR-30a was significantly reduced in U251 cells treated with TMZ in a concentration-dependent manner, when compared with the control group. Therefore, miR-30a may be involved in TMZ-induced autophagy in U251 cells.
A

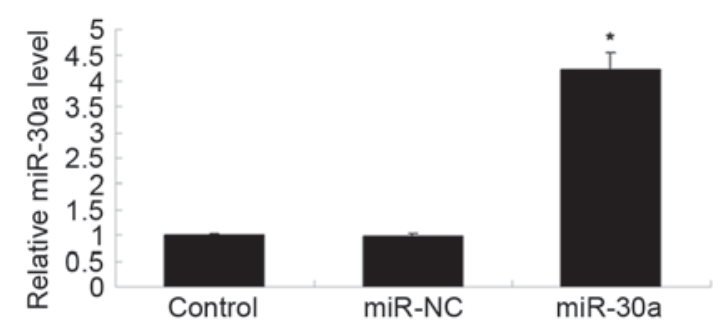

B

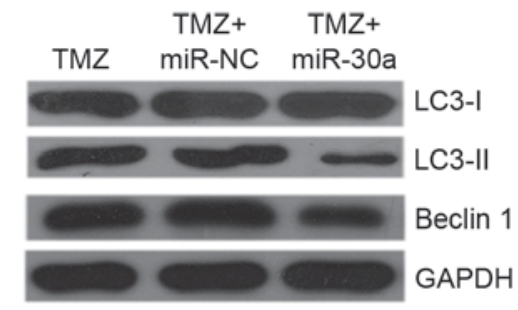

- TMZ घ TMZ+miR-NC $\square \mathrm{TMZ}+\mathrm{miR}-30 \mathrm{a}$

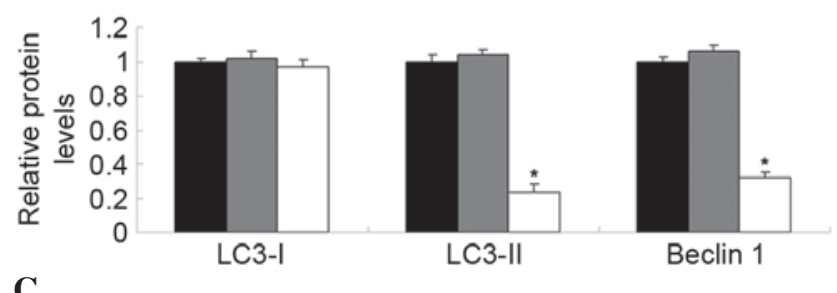

C

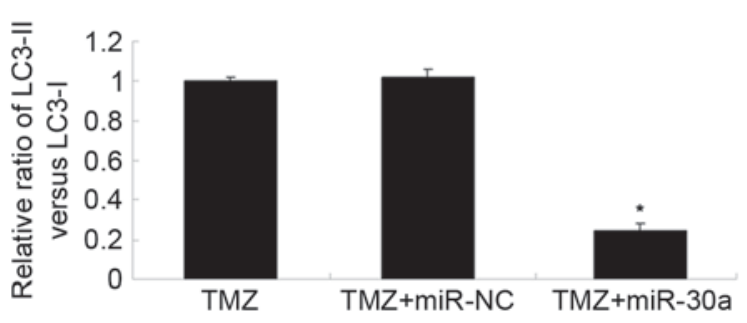

Figure 3. Restoration of miR-30a level suppresses TMZ-induced autophagy in U251 cells via inhibition of beclin 1. (A) Reverse transcription-quantitative polymerase chain reaction was conducted to examine the relative miR-30a levels in U251 cells transfected with miR-30a mimic or miR-NC, respectively. (B) Western blot analysis was conducted to determine the levels of autophagy-related proteins in the transfected U251 cells treated with TMZ (30 $\mu \mathrm{g} / \mathrm{ml})$. (C) The ratio of LC3-II to LC3-I was calculated. Non-transfected U251 cells treated with TMZ were used as the control. ${ }^{*} \mathrm{P}<0.05$ vs. TMZ. TMZ, temozolomide; miR-NC, scrambled miR.

Elevation of miR-30a level suppresses TMZ-induced autophagy in U251 cells via inhibition of beclin 1. To further determine the role of miR-30a in the beclin 1-mediated autophagy of TMZ-treated glioblastoma cells, U251 cells were transfected with miR-30a mimic or scrambled miR mimic as a negative control prior to TMZ treatment. As shown in Fig. 3A, transfection with miR-30a mimic markedly increased the miR-30a levels, while transfection with scrambled miR mimic had no effect on the miR-30a level, when compared with that of the control group. U251 cells were then treated with TMZ (30 $\mu \mathrm{g} / \mathrm{ml}$ ) for $6 \mathrm{~h}$, and expression levels of autophagy markers were examined using western blot assay. As shown in Fig. 3B, the protein levels LC3-II and beclin 1 were lower in U251 cells transfected with miR-30a mimic, compared with the control. Moreover, the ratio of LC3-II to LC3-I was reduced in miR-30a-overexpressing U251 cells treated with TMZ, 
A

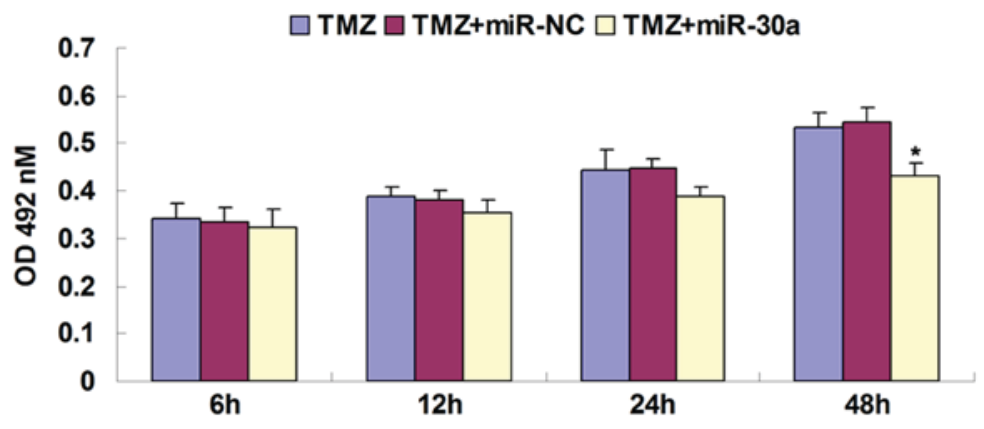

B
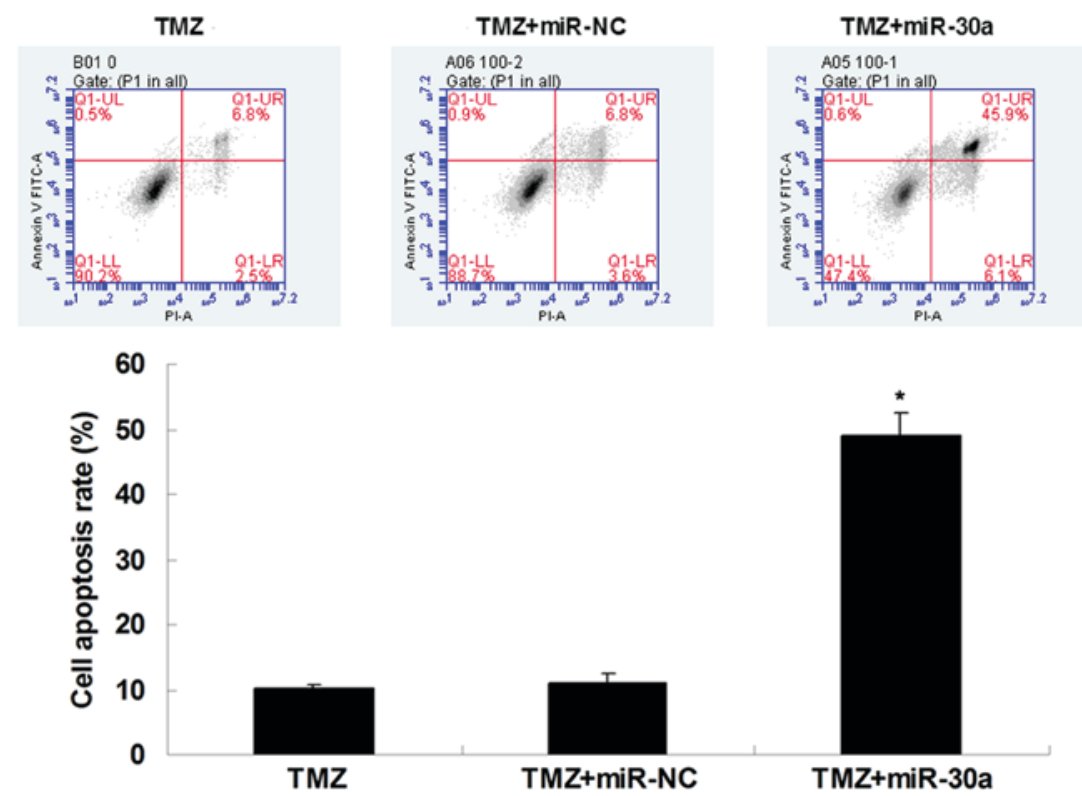

Figure 4. Overexpression of miR-30a increases the cytotoxicity of TMZ to U251 cells. (A) MTT assay was performed to examine the proliferation of TMZ $(30 \mu \mathrm{g} / \mathrm{ml})$-treated U251 cells transfected with miR-30a mimic or miR-NC, respectively. (B) Flow cytometry was conducted to examine cell apoptosis. Non-transfected U251 cells treated with TMZ $(30 \mu \mathrm{g} / \mathrm{ml})$ were used as control. "P<0.05 vs. TMZ. TMZ, temozolomide; miR-NC, scrambled miR.

compared with the control cells (Fig. 3C). These findings indicate that overexpression of miR-30a significantly suppressed TMZ-induced autophagy in U251 glioblastoma cells.

Overexpression of miR-30a increases the cytotoxicity of TMZ to U251 cells. Whether miR-30a upregulation could promote the TMZ-induced inhibition of proliferation and/or TMZ-induced apoptosis of U251 cells was investigated. As shown in Fig. 4A, MTT assay results showed that the overexpression of miR-30a significantly suppressed the proliferation of TMZ (30 $\mu \mathrm{g} / \mathrm{ml})$-treated U251 cells compared with the control group. However, transfection with scrambled miR mimic caused no difference in the proliferation of U251 cells, when compared with the control group. These data indicate that overexpression of miR-30a increased the TMZ-induced inhibition of glioblastoma cell proliferation. It was further observed that an upregulated level of miR-30a also led to a significant increase in the apoptosis of TMZ-treated U251 cells, when compared with the control group (Fig. 4B), indicating that upregulation of miR-30a also promoted the TMZ-induced apoptosis of glioblastoma cells. Together, these results indicate that overexpression of miR-30a increased the cytotoxicity of TMZ to glioblastoma U251 cells.
Beclin 1 is a direct target gene of miR-30a in U251 cells. Finally, the relationship between miR-30a and beclin 1 in U251 cells was examined. Bioinformatic prediction data indicated that beclin 1 is a direct target gene of miR-30a (Fig. 5A). To clarify this relationship, WT or MUT BECN1 3'-UTR was cloned into the pmirGLO vector, downstream of the firefly luciferase coding region, to generate pMIR-WT BECN1 and pMIR-MUT BECN1, respectively (Fig. 5B and C). A luciferase reporter assay was further conducted in the U251 cells. As indicated in Fig. 5D, co-transfection with pMIR-WT BECN1 and miR-30a mimic led to a significant reduction in luciferase activity; however, co-transfection with pMIR-MUT BECN1 and miR-30a mimic caused no change in luciferase activity, indicating that miR-30a could directly bind to the 3'-UTR of beclin 1 mRNA in U251 cells. These results demonstrate that beclin 1 is a direct target of miR-30a in U251 cells, and suggest that the role of miR-30a in TMZ-induced autophagy involves the mediation of beclin 1 expression in $\mathrm{U} 251$ cells.

\section{Discussion}

Autophagy has been demonstrated to be important not only in the recirculation of degraded components to sustain metabolic 
A

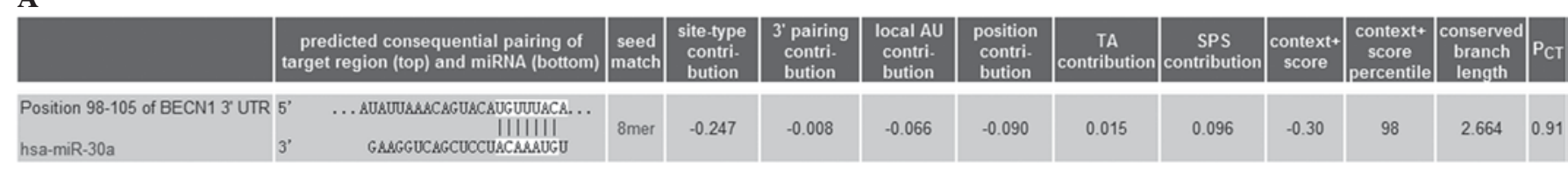

B

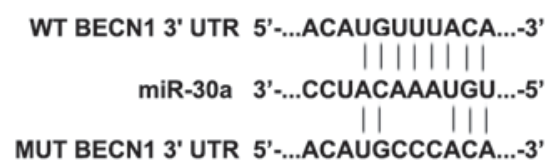

C

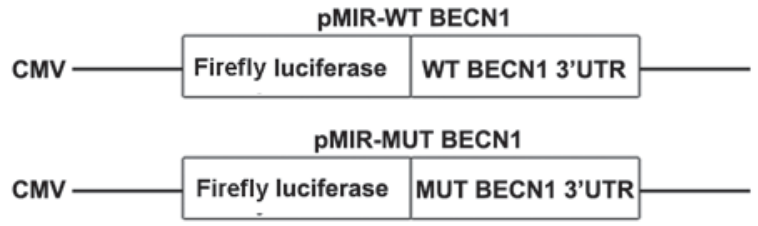

D

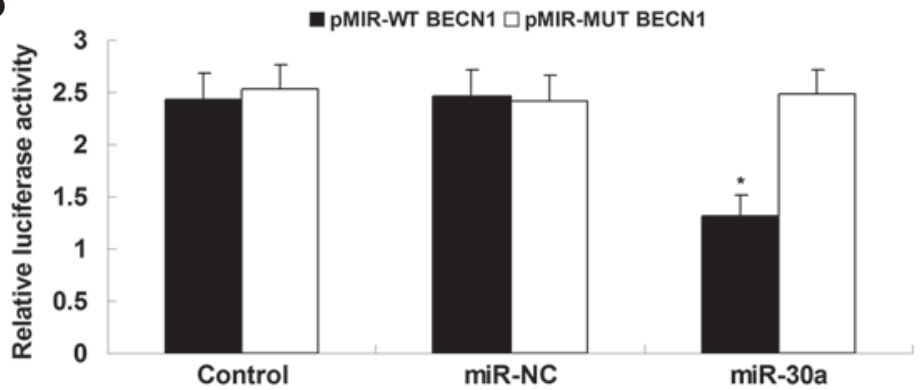

Figure 5. Beclin 1 is a direct target of miR-30a in U251 cells. (A) Bioinformatic prediction data indicated that beclin 1 was a direct target gene of miR-30a. (B and C) WT or MUT BECN1 3'-UTR was cloned downstream of the firefly luciferase coding region of the pmirGLO ${ }^{\mathrm{TM}}$ vector, to form pMIR-WT BECN1 and pMIR-MUT BECN1, respectively. (D) U251 cells were co-transfected with pMIR-WT BECN1 or pMIR-MUT BECN1 vector and miR-30a mimic or miR-NC, and pRL-TK plasmid for internal normalization, respectively. Luciferase reporter assay data showed that co-transfection with pMIR-WT BECN1 and miR-30a mimic significantly decreased the luciferase activity; however, co-transfection with pMIR-MUT BECN1 and miR-30a mimic caused no change in luciferase activity. Control cells were transfected with pMIR-WT BECN1 or pMIR-MUT BECN1 vector and the pRL-TK plasmid. "P<0.05 vs. control. WT, wild type; MUT, mutant; BECN1, beclin 1; UTR, untranslated region; miR-NC, scrambled miR mimic.

homoeostasis, but also in the prevention of the toxic accumulation of damaged components (9). Elevated autophagy has been found in a variety of tumor cells subjected to certain stresses, including chemotherapy drug treatment, and it has been well established that autophagy can lead to the chemotherapy resistance of various human cancers (11-13). Therefore, inhibition of chemotherapy drug-induced autophagy appears to be a promising strategy for enhancing the efficiency of chemotherapy in human cancers. In the present study, it was found that treatment with TMZ not only inhibited glioblastoma cell proliferation and induced apoptosis in a concentration-dependent manner, but also induced the activation of autophagy, which might attenuate the cytotoxicity of TMZ to U251 glioblastoma cells. The study further demonstrated that overexpression of miR-30a inhibited TMZ-induced autophagy by targeting beclin 1 , and increased the TMZ-induced inhibition of cell proliferation in addition to TMZ-induced cell apoptosis in U251 cells.

TMZ has been widely used for the treatment of many types of human cancers, including glioblastoma. It has been reported that the antitumor activity of TMZ is achieved through DNA damage via induction of loop structures as well as DNA condensation (24). However, treatment with TMZ often leads to tumor cell resistance to TMZ due to autophagy (13). Indeed, in the present study, treatment with TMZ not only caused cytotoxicity but also increased glioblastoma cell autophagy, as demonstrated by the upregulated protein levels of beclin 1 and LC3-II, which was probably caused by the downregulation of miR-30a.
miR-30a has been found to play a key role in multiple types of human cancers, mainly acting as a tumor suppressor.For instance, Fu et al reported that miR-30a suppressed breast cancer cell proliferation and migration by targeting Eya2 (25). Zhong et al found that miR-30a suppressed cell migration and invasion through downregulation of phosphatidylinositol-4,5-bisphosphate 3-kinase catalytic subunit $\delta$ in colorectal carcinoma (26). Several studies have indicated that miR-30a has suppressive effects on autophagy in different cell types via the direct targeting of beclin 1 (27-29). For instance, Wang et al found that inhibition of miRNA-30a alleviated cerebral ischemic injury by increasing beclin 1-mediated autophagy (30). In the present study, it was found that treatment with TMZ induced a significant reduction in miR-30a level in a dose-dependent manner in U251 cells. In addition, beclin 1 has been demonstrated to function as a key inducer of autophagy (31), and has been found to participate in the regulation of tumorigenesis (32). In the present study, the protein level of beclin 1 was significantly increased in U251 cells treated with TMZ, while overexpression of miR-30a inhibited the TMZ-induced upregulation of beclin 1 in U251 cells. As beclin 1 was found to be a direct target of miR-30a in U251 cells, it is suggested that the suppressive effect of miR-30a on TMZ-induced autophagy is achieved through the direct mediation of beclin 1 expression in U251 cells.

miR-30a has been found to sensitize tumor cells to several different chemotherapy drugs. Zou et al (21) found that miR-30a sensitized tumor cells to cisplatin via the suppression of beclin 1-mediated autophagy. Furthermore, knockdown of 
miR-30a has been demonstrated to increase the expression of beclin 1 and inhibit imatinib-induced cytotoxicity (23). In the present study, it was found that elevation of the miR-30a level in TMZ-treated U251 cells notably increased the cytotoxicity of TMZ to tumor cells, as demonstrated by the reduced cell proliferation as well as the increased cell apoptosis.

In conclusion, the present study demonstrated that treatment with TMZ induced an activation of autophagy as well as a downregulation of miR-30a, while overexpression of miR-30a inhibited the expression of beclin 1, and thus suppressed TMZ-induced autophagy in U251 cells. Inhibition of autophagy by the elevation of miR-30a expression enhanced the cytotoxicity of TMZ to U251 cells. Based on these findings, it is suggested that autophagy may be a promising target for the treatment of TMZ-resistant tumors.

\section{Acknowledgements}

This study was supported by the National Natural Science Foundation of China (grant no. 81201740) and as a project of the Hunan Province Science and Technology Department (grant no. 2012FJ6075).

\section{References}

1. Goodenberger ML and Jenkins RB: Genetics of adult glioma. Cancer Genet 205: 613-621, 2012.

2. Stewart LA: Chemotherapy in adult high-grade glioma: A systematic review and meta-analysis of individual patient data from 12 randomised trials. Lancet 359: 1011-1018, 2002.

3. Zhu VF, Yang J, Lebrun DG and Li M: Understanding the role of cytokines in glioblastoma multiforme pathogenesis. Cancer Lett 316: 139-150, 2012.

4. Sathornsumetee S, Reardon DA, Desjardins A, Quinn JA, Vredenburgh JJ and Rich JN: Molecularly targeted therapy for malignant glioma. Cancer 110: 13-24, 2007.

5. Pulkkanen KJ and Yla-Herttuala S: Gene therapy for malignant glioma: Current clinical status. Mol Ther 12: 585-598, 2005.

6. Messaoudi K, Clavreul A and Lagarce F: Toward an effective strategy in glioblastoma treatment. Part II: RNA interference as a promising way to sensitize glioblastomas to temozolomide. Drug Discov Today 20: 772-779, 2015.

7. Citıslı V, Dodurga Y, Eroğlu C, Seçe M, Avcı ÇB and Şatıroğlu-Tufan NL: Temozolomide may induce cell cycle arrest by interacting with URG4/URGCP in SH-SY5Y neuroblastoma cells. Tumour Biol 36: 6765-6772, 2015.

8. Li G, Zhang H, Liu Y, Kong L, Guo Q and Jin F: Effect of temozolomide on livin and caspase-3 in U251 glioma stem cells. Exp Ther Med 9: 744-750, 2015.

9. Su Z, Yang Z, Xu Y, Chen Y and Yu Q: MicroRNAs in apoptosis, autophagy and necroptosis. Oncotarget 6: 8474-8490, 2015.

10. Wang QW, Wang Y, Wang T, Zhang KB, Jiang CY, Hu FF, Yuan Y, Bian JC, Liu XZ, Gu JH and Liu ZP: Cadmium-induced autophagy promotes survival of rat cerebral cortical neurons by activating class III phosphoinositide 3-kinase/beclin-1/B-cell lymphoma 2 signaling pathways. Mol Med Rep 12: 2912-2918, 2015.

11. Kong Q, Xu LH, Xu W, Fang JP and Xu HG: HMGB1 translocation is involved in the transformation of autophagy complexes and promotes chemoresistance in leukaemia. Int J Oncol 47: 161-170, 2015.

12. Sehgal AR, Konig H, Johnson DE, Tang D, Amaravadi RK, Boyiadzis M and Lotze MT: You eat what you are: Autophagy inhibition as a therapeutic strategy in leukemia. Leukemia 29: 517-525, 2015.

13. Yang MC, Loh JK, Li YY, Huang WS, Chou CH, Cheng JT, Wang YT, Lieu AS, Howng SL, Hong YR and Chou AK: $\mathrm{Bcl} 2 \mathrm{~L} 12$ with a BH3-like domain in regulating apoptosis and TMZ-induced autophagy: A prospective combination of ABT-737 and TMZ for treating glioma. Int J Oncol 46: 1304-1316, 2015.
14. Zhou Y, Wang HD, Zhu L, Cong ZX, Li N, Ji XJ, Pan H, Wang JW and Li WC: Knockdown of Nrf2 enhances autophagy induced by temozolomide in U251 human glioma cell line. Oncol Rep 29: 394-400, 2013.

15. Fu J, Liu ZG, Liu XM, Chen FR, Shi HL, Pangjesse CS, $\mathrm{Ng} \mathrm{HK}$ and Chen ZP: Glioblastoma stem cells resistant to temozolomide-induced autophagy. Chin Med J (Engl) 122: 1255-1259, 2009.

16. Ambros V: The functions of animal microRNAs. Nature 431: 350-355, 2004.

17. BartoszewskaS, Kochan K,Piotrowski A,Kamysz W,OchockaRJ, Collawn JF and Bartoszewski R: The hypoxia-inducible miR-429 regulates hypoxia-inducible factor-1 $\alpha$ expression in human endothelial cells through a negative feedback loop. FASEB J 29: 1467-1479, 2014.

18. Zhou S, Zhang P, Liang $\mathrm{P}$ and Huang $\mathrm{X}$ : The expression of miR-125b regulates angiogenesis during the recovery of heat-denatured HUVECs. Burns 41: 803-811, 2015.

19. He R, Peng J, Yuan P, Xu F and Wei W: Divergent roles of BECN1 in LC3 lipidation and autophagosomal function. Autophagy 11: 740-747, 2015

20. Zheng B, Zhu H, Gu D, Pan X, Qian L, Xue B, Yang D, Zhou J and Shan Y: MiRNA-30a-mediated autophagy inhibition sensitizes renal cell carcinoma cells to sorafenib. Biochem Biophys Res Commun 459: 234-239, 2015.

21. Zou Z, Wu L, Ding H, Wang Y, Zhang Y, Chen X, Chen X, Zhang CY, Zhang Q and Zen K: MicroRNA-30a sensitizes tumor cells to cis-platinum via suppressing beclin 1-mediated autophagy. J Biol Chem 287: 4148-4156, 2012.

22. Yu Y, Yang L, Zhao M, Zhu S, Kang R, Vernon P, Tang D and Cao L: Targeting microRNA-30a-mediated autophagy enhances imatinib activity against human chronic myeloid leukemia cells. Leukemia 26: 1752-1760, 2012.

23. Livak KJ and Schmittgen TD: Analysis of relative gene expression data using real-time quantitative PCR and the 2(-Delta Delta C(T)) Method. Methods 25: 402-408, 2001.

24. Kondo N, Takahashi A, Mori E, Noda T, Zdzienicka MZ, Thompson LH, Helleday T, Suzuki M, Kinashi Y, Masunaga S, et al: FANCD1/BRCA2 plays predominant role in the repair of DNA damage induced by ACNU or TMZ. PLoS One 6: e19659, 2011.

25. Fu J, Xu X, Kang L, Zhou L, Wang S, Lu J, Cheng L, Fan Z, Yuan B, Tian P, et al: Mir-30a suppresses breast cancer cell proliferation and migration by targeting Eya2. Biochem Biophys Res Commun 445: 314-319, 2014.

26. Zhong M, Bian Z and Wu Z: Mir-30a suppresses cell migration and invasion through downregulation of PIK3CD in colorectal carcinoma. Cell Physiol Biochem 31: 209-218, 2013.

27. Zhu H, Wu H, Liu X, Li B, Chen Y, Ren X, Liu CG and Yang JM: Regulation of autophagy by a beclin 1-targeted microRNA, miR-30a, in cancer cells. Autophagy 5: 816-823, 2009.

28. Huang J, Huang C, Luo Y, Liu S and Chen X: Role of MiR-30a in cardiomyocyte autophagy induced by Angiotensin II. J Renin Angiotensin Aldosterone Syst 16: 1-5, 2015.

29. Chen Z, Wang T, Liu Z, Zhang G, Wang J, Feng S and Liang J: Inhibition of autophagy by MiR-30A induced by mycobacteria tuberculosis as a possible mechanism of immune escape in human macrophages. Jpn J Infect Dis 68: 420-424, 2015.

30. Wang P, Liang J, Li Y, Li J, Yang X, Zhang X, Han S, Li S and Li J: Down-regulation of miRNA-30a alleviates cerebral ischemic injury through enhancing beclin 1-mediated autophagy. Neurochem Res 39: 1279-1291, 2014.

31. He Y, Zhao X, Subahan NR, Fan L, Gao J and Chen H: The prognostic value of autophagy-related markers beclin-1 and microtubule-associated protein light chain 3B in cancers: A systematic review and meta-analysis. Tumour Biol 35: 7317-7326, 2014.

32. Elgendy M, Ciro M, Abdel-Aziz AK, Belmonte G3, Dal Zuffo R, Mercurio C, Miracco C, Lanfrancone L, Foiani M and Minucci S: Beclin 1 restrains tumorigenesis through Mcl-1 destabilization in an autophagy-independent reciprocal manner. Nat Commun 5: 5637,2014

This work is licensed under a Creative Commons Attribution-NonCommercial-NoDerivatives 4.0 International (CC BY-NC-ND 4.0) License. 